\title{
LES POISSONS MIGRATEURS DANS L'ECONOMIE PISCICOLE DU SUD-OUEST
}

\author{
par M. R. VIBERT \\ Inspecteur des Eaux et Farsts, \\ Chêt de la $3^{e}$ Région piscicole.
}

\section{I. - Importance de la pÊChe fluviale}

Pour déterminer l'importance du domaine piscicole constitué par les eaux douces de France et l'estimer à sa juste valeur, il est nécessaire d'en connaître l'étendue et de déduire de cêtte dernière le poids de poisson qu'il produit.

L est très difficile, pour ne pas dire impossible, dans l'état actuel de nos connaissances, de donner, sur l'importance de notre domaine piscicole, des précisions pouvant échapper à la critique.

Si la longueur des fleuves navigables et flottables faisant partie du domaine public est connue avec une approximation suffisante, il $n$ 'en est pas de mème des cours d'eau du domaine privé qui constituent la majeure partie du réseau fluvial français et pour lesquels les chifires publiés varient de 140.000 à 400.000 kilomètres, suivant que les auteurs n'ont considéré que les cours d'eau ayant une certaine importance ou ont tenu compte du moindre ruisseau.

M. I'Inspecteur des Eaux et Forêts Charpy, Chef de la $1^{\text {ra }}$ Région piscicole, a eu le mérite, dans un travail récent (1), de clarifier la question en résumant les travaux statistiques antérieurs et en proposant à son tour les chiffres suivants qui, selon ses propres termes, " q'ont pas une valeur absolue mais simplement une valeur critique " :

- Fleuves et rivières navigables et flottables : 11.265 kilomètres.

- Canaux : 2.310 kilomètres.

- Rivières non navigables ni flottables : 258.574 kilomètres.

- Lacs du domaine public : 50.000 hectares.

- Etangs privés : 100.000 hectares.

Si l'estimation de la longueur de nos rivières a conduit, comme nous l'avons vu, à des chiffres variant du simple au double, l'évaluation de la

(1) Voir Bulletin - Juillet-Décembre 1941, p. 38, - no 124, Janvier-Mars 1942, p. 80 . 
production de notre plan d'eau national a conduit à des chiffres variant

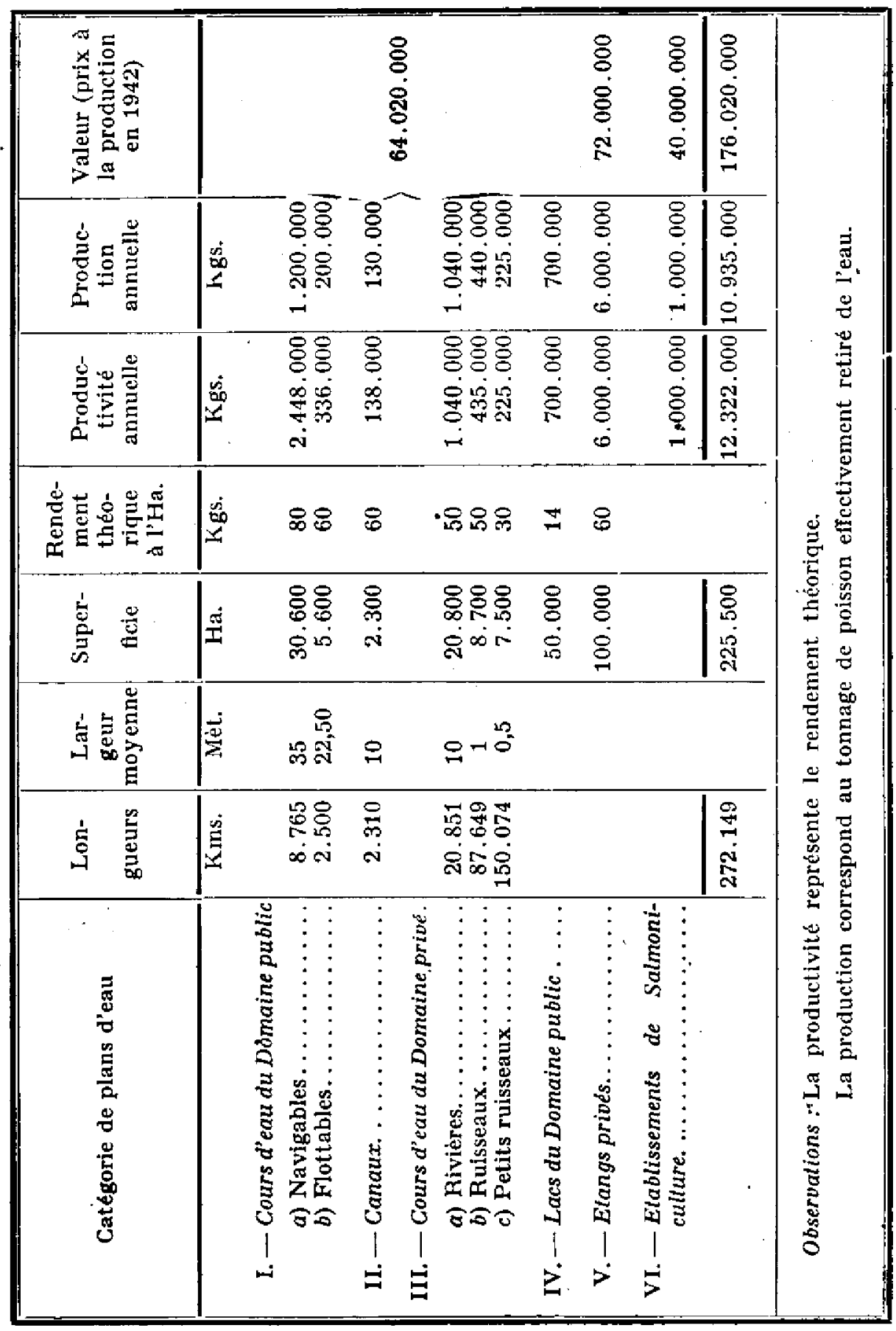

en chiffre rond de 67.000 tonnes (DE Cafdaillac-de-Samt-Paul 1902) à 9.000 tonnes (Florian-Cnardon 1936). Il est vrai que, de 1902 à 1936, la 
richesse de nos rivières a, selon toute vraisemblance, singulièrement diminué, surtout pour les Poissons migrateurs.

Un plan d'eau d'un hectare cst capable, tout comme une terre à blé, de produire une quantité déterminée de poisson en rapport avec sa richesse en nourriture, richesse que le Professeur LÉGen a appelée " coefficient biogénique ". Nous ne nous attarderons pas sur la mesure de ce coefficient qui varie de I à $\mathrm{X}$, ni sur son emploi par les Pisciculteurs, mentionnons simplement qu'un plan d'eau d'un hectare peut, selon ses caractéristiques, produire annuellement 10 à 100 kilos de Salmonidés ou 20 à 200 kilos de Poissons blancs.

C'est en appliquant ces notions récentes à l'étendue des plans d'eau des différentes catégories de rivières, en attribuant à chacun d eux les largeurs moyennes déjả énoncées par GobIs, en 1889 , que M. l'Inspecteur CharpY a établi en 1941 ses estimations de la productivité et de la production annuelle du domaine piscicole français qui peuvent se résumer dans le tableau ci-contre :

Nous $n$ 'avons aucun moyen à l'heure actuelle d'infirmer ou de confirmer ces chiffres qui ne sont d'ailleurs, selon M. l'Inspecteur Chafpy, "qu'une base de départ pour un travail plus poussé ", mais notre impression personnelle est qu'il y a une sous-estimation dans la fixation à 35 mètres de la largeur moyenne de nos cours d'eau navigables, sous-estimation qui a évidemment sa répercussion dans l'évaluation de la production.

Ces chiffres ne donnent une idée que de la valeur économique directe du poisson capturé. Il y a lieu de tenir compte également des répercussions indirectes sur l'Economie générale et la vie du pays : - importance de la péche au point de vue social, - influence sur l'industrie hôtelière et le tourisme - développement intense depuis trente ans des fabrications d'articles de péche (300 millions de francs en $1936 !$ ).

Il faut, cependant, remarquer que les estimations de la productivité et de la production, à partir de la capacité biogénique de nos rivières, ne tiennent aucun compte de nos poissons migrateurs tels que le Saumon, l'Esturgeon, l'Alose..., qui ne se nourrissent en eau douce que pendant leur tout jeune åge, vont grandir en mer et reviennent ensuite comme adultes dans nos cours d'eau où ils observent un jeûne complet avant de se faire capturer ou de se reproduire.

Divers auteurs nous ont déjà appris qu'il fallait considérer ces captures de migrateurs comme un "superdividende ". Quelle est son importance en regard du " dividende " résultant des chiffres de M. l'Inspecteur des Eaux et Forêts Charpy p' C'est là une incơnnue à peu près totale dont nous allons nous efiorcer de soulever tout au moins une partie du voile. 


\section{II. - Poissons migrateuns fréquentant les eaux douces DU SUD-OUEBT DE LA FHANCE}

\section{A. - Principales espèces.}

Le Saumon. - A tout seigneur tout honneur. Examinons, tout d'abord, très rapidement, les caractéristiques biologiques de ce " poisson royal $\mathrm{n}$. Ce magnifique représentant de la famille des Salmonidés était si commun en France, il n y a pas plus d'un siècle, que des contrats de louage de domestiques, devenus fameux, prescrivaient que ces derniers ne devaient pas être nourris de Saumon plus de deux ou trois fois par semaine, et cela dans presque toutes les campagnes riveraines de nos petits fleuves côtiers picards, normands, bretons, de la Seine et de ses principaux affluents, de la Loire grossie de l'Allier, de la Vienne et de la Creuse, de la presque totalité des bassins de la Garonne et de la Dordogne, des gaves pyrénéens et de la Nive.

Notre Saumon du Sud-Ouest peut atteindre une bonne vingtaine de kilos. Son poids moyen est de 10 kilos environ. De l'avis des pècheurs sportifs anglais, il ne le cède en rien aux plus beaux Saumons d'Ecosse.

La ponte a lieu en Décembre-Janvier, dans les eaux froides, aérées et très pures de têtes de bassins. L'alevin reste en moyenne deux ans dans ces régions, descend à la mer en véritables banç, y reste en moyenne trois ans puis, adulte, remonte pour frayer dans les hautes vallées où il a passé son enfance.

L'Esturgeon. - Contrairement à l'opinion courante, ce n'est pas un poisson cantonné uniquement en Russie et en Roumanie. Le Rhône et l'Adour en ont encore quelques spécimens, en Gironde et en Basse-Garonne, il est un des poissons les plus communs. Les mâles atteignent 20 à 30 kilos ; les femelles peuvent dépasser 200 kilos.

La ponte a lieu en Garonne dans les grands courants, par 6 à 8 mètres de fond, entre La Réole et Agen, dans l'Adour au voisinage du confluent des Gaves réunis. Les alevins âgés seulement de quelques mois descendent dans l'estuaire. Ils atteignent là une taille de 70 centimètres à I mètre, se trouvent souvent en bancs serrés sur les graviers, principalement en Gironde, où ils donnent lieu au plus stupide des braconnages. Ils descendent ensuite en mer pour atteindre leur complet développement, puis durant les mois de Juin-Juillet en Garonne, Aout dans l'Adour, remontent pour frayer. Les cufs sont livrés comme caviar aux maisons "Prinier " et "Volga ".

L'Alose. dont la grande espèce "Alosa-Alosa " atteint $3 \mathrm{kilos}_{\mathbf{l}}$ a dans IAdour et la Garonne, ainsi que dans la Dordogne, les mêmes "tenues" que l'Esturgeon. Ses migrations sont les mêmes, sauf toutefois, que les jeunes alevins de quelques mois qui descendent à la mer n'éprouvent pas le besoin de faire une halte prolongée dans les estuaires. 
La Lamproie marine peut atteindre un mètre, naît en eau douce dans la partie basse des vallées, descend à la mer pour s'y développer, puis revient au printemps pour se reproduire.

Les Mulets peuvent atteindre en mer, à maturité, 70 à 80 centimètres. Ils naissent en mer. Les jeunes s'avancent au printemps dans les eaux saumatres, et parfois assez loin eu eau douce, puis redescendent à l'automne pour aller terminer leur croissance en mer.

Le Flet, qui peut atteindre 50 centimètres, fréquente les estuaires, mais remonte souvent assez haut en eau douce.

Nous la isserons ici volontairement de côté les migrateurs comme l'Anguille è ia Lamproie de rivière qui prennent tout ou partie de leur nourritırc en eau douce et qui ne peuvent, à ce titre, figurer dans le a superdividende " de nos cours d'equ.

\section{B. - Importance des captures.}

L'exploitation directe des lots de pêche sur nos cours d'eau n'ayant à notre connaissance jamais été tentée à ce jour par l'Administration, on ne peut baser des estimations que sur des enquêtes auprès des pêcheurs professionnels... c'est dire les difficultés auxquelles on se heurte immanquablement. En temps normal, ces derniers déclarent toujours qu'il ne prennent rien ou presque, de peur de voir augmenter le loyer de leurs lots; dans les circonstances actuelles, leur méfiance est encore centuplée du fait que, le plus souvent, le ravitaillement officiel ne perçoit pratiquement qu'une part infime de leurs prises.

Cette méfiance sera vraisemblablement susceptible de s'atténuer, le jour où certains de ces professionnels se rendront compte que le Service de la Pêche s'efforce de toul mettre en ouvre pour enrayer la raréfaction progressive de ces poissons migrateurs dont la capture les fait vivre, et que ce service a besoin d'une sérieuse documentation pour pouvoir agir en connaissance de cause. Quelques présidents de groupements professionnels ont bien voulu nous faire confiance, et nous communiquer les résultats de leur pèche... à titre confidentiel. On voudra bien, en conséquence, nous excuser de ne pas toujours préciser les secteurs auxquels se rapprochent les chiffres qu'il nous sera possible de donner.

Les prises de Saumon, d'après les enquêtes menéen dans les Basses-Pyrénées depuis quelque vingt ans, oscillent pour ce seul Département entre 10 et 20.000 Saumons par an, soit une moyenne de 15.000 , à 10 kilogrammes pièce : 150.000 kilogrammes à 100 francs à la production :

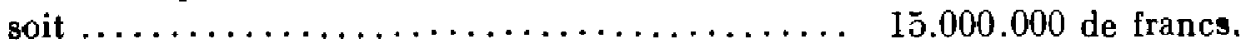

Pour ce qui est de l'Esturgeon, le régime actuel d'économie dirigée va nous permettre de montrer son importance dans notre Sud-Ouest : Les deux 
maisons "Prunier " et "Yolga ", seules détentrices d'une licence de fabricatjon de caviar, ont ramassé ces dernières années, rien que pour le Département de la Gironde, une moyenne de 2.000 kilogrammes d'cufs d Esturgeons par an (chiffre enregistré par le "Contrôle des Conserves et SousProduits ", organe de l'Office Scientifique et Technique des Pêchcs maritimes). Ces cufs sont payés 1.000 francs le kilo au pècheur,

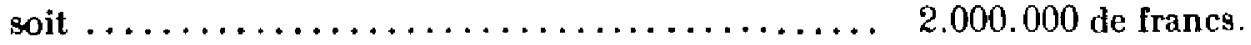

Ces 2.000 kilogrammes d'cufs (10\% du poids de la femelle) représentent 20.000 kilogrammes d'Esturgeons femelles et 200.000 kilogrammes d'Esturgeons mảles (vingt fois plus nombreux et deux fois plus petits en moyenne). A ces chiffres doivent s'ajouter au minimum 100.000 kilogrammes de jeunes Esturgeons de quelques décimètres, stupidement détruits sur les hauts fonds de la Gironde par les Inscrits maritimes qui tuent ainsi en enfance leur "Poisson aux oufs d'or".

Soit un minimum en chiffres ronds, pour le seul Département de la Gironde de : 300.000 kilogrammes à 25 francs ...... 7.500 .000 francs.

Un premier essai de ramassage des oufs d'Esturgeon dans l'Adour, dans les Basses-Pyrénées et les Landes, a élé tenté en 1943, il a rapporté 143 kilogrammes d'œufs à 1.000 francs .............. 143.000 francs.

Chiffre correspondant approximativement à 14.300 kilogrammes d'Esturgeons à 25 francs $\ldots \ldots \ldots \ldots \ldots \ldots \ldots \ldots \ldots \ldots \ldots \ldots \ldots$ francs.

Soit, pour l'ensemble : 500.000 francs.

Le ramassage doit être également tếnté dans le Lot-et-Garonne.

L'Alose n'a fait l'objet, jusqu'à présent, d'aucune centralisation effective des prises, et nous sommes contraints de nous contenter pour le moment de résultats trop fragmentaires encore, mais qui s'avèrent être des plus intéressants, malgré les très mauvaises pêches faites sur ce poisson depuis quelques années. En 1943, tel pêcheur sur un lot de 9 kilomètres en a capturé 18.000 kilogrammes. Tel secteur de 30 kilomètres est annoncé pour 48.000 kilogrammes. Tel Département pour 50.000 kilogrammes. Nous pouvons donc, dès à présent, envisager pour les Départements du Sud-Ouest une production de 150 à 300.000 kilogrammes, soit une moyenne de : 200.000 kilogrammes à 25 francs... 5.000 .000 de francs.

Nous n'avons encore aucun renseignement sérieux sur l'importance des captures de Lamproies marines.

Quant aux captures de Mulets et de Flets, elles équivalent les prises d'Aloses. Nous ne les indiquons néanmoins que pour mémoire, ces deux poissons n'étant que des migrateurs très passagers, qui ne se reproduisent pas en 'eau douce, qui n'y observent pas un jeûne absolu comme les précédents et ne doivent pas, en conséquence, être incorporés en totalité au " superdividende".

En résumé, en attendant que des études plus fouillées nous apportent des précisions complémentaires, nous pouvons admettre comme ordre de 
grandeur actuel du "superdividende " des bassing de la Garonne et de l'Adour les chiffres suivants:

\begin{tabular}{|c|c|c|}
\hline ESPĖCES & POIDS & VALEUR \\
\hline \multirow[t]{2}{*}{$\begin{array}{l}\text { Saumons } \ldots \ldots \ldots \ldots \ldots \ldots \ldots \ldots \\
\text { Aloses. } \ldots \ldots \ldots \ldots \ldots \ldots \ldots \ldots \ldots \ldots \\
\text { Esturgeons } \ldots \ldots \ldots \ldots \ldots \ldots \ldots \ldots \ldots \\
\text { Caviars. } \ldots \ldots \ldots \ldots \ldots \ldots \ldots\end{array}$} & $\begin{array}{l}150.000 \text { kỉos. } \\
200.000- \\
314.300- \\
2.143-\end{array}$ & $\begin{array}{r}15.000 .000 \mathrm{fr} . \\
5.000 .000- \\
7.857 .000- \\
2.143 .000-\end{array}$ \\
\hline & TotaL...... & $30.000 .000 \mathrm{fr}$ \\
\hline
\end{tabular}

Ces migrateurs ont d'autant plus d'importance que ce sont tous des poissons de choix à prix unitaire élevé. De l'arrêté ministériel du 23 Novembre 1943, nous pouvons, en effet, retenir les termes de comparaison suivants :

\begin{tabular}{|c|c|}
\hline ESPËCES & $\begin{array}{l}\text { PRIX MOYENS A LA PRODUCTION } \\
\text { (en chiffre rond) }\end{array}$ \\
\hline 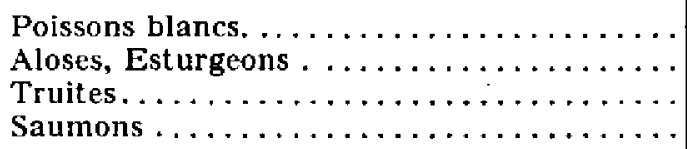 & $\begin{array}{l}10 \mathrm{fr} . \text { le kilo. } \\
25 \mathrm{fr} \quad- \\
50 \mathrm{fr} \text { - } \\
100 \mathrm{fr} .\end{array}$ \\
\hline
\end{tabular}

Des études de M. l'Inspecteur Charpy, il ressort, par ailleurs, que le dividende correspondant à la production annuelle des véritables poissons d'eau douce de l'ensemble du réseau fluvial, y compris les lacs du domaine public, peut être évalué à 3.935 .000 kilogrammes, d'une valeur de 64.020 .000 francs. La part revenant aux cours d'eau navigables (les seuls en gros fréquentés par les grands migrateurs) se chiffre a 1.200 .000 kilogrammes, ce qui à 10 francs le kilogramme, représente un dividende de $12.000 .000(1)$.

(1) Les prix unitaires retenus par M. l'Inspecteur ChaRPy dans son étude citée précédemment étaient de 12 francs pour le poisson blanc, de 40 francs pour la truite :

9.335 .000 kilos de poisson blanc à 12 francs. . . . . 112.020 .000 fr. "

1.600 .000 kilos de truite a 40 francs. . . . . . . . . 64.000 .000 fr. "

Total....... $\overline{176.020 .000 \text { fr. }}$

L'adoption des prix unitaires actuels (10 et 50 fr.) conduirait à un total de 179.350.000 francs. La différence ne vaut pas la peine d'être notée. Aucune péréquation quant au prix unitaire n'est nécessaire pour comparer le rendement des péches des migrateurs (superdividende) avec celui de la pèche de nos poissons d'eau douce (dividende). 
La $3^{\circ}$ Région piscicole peut prétendre au maximum en ce qui la concerne (18 Départements touchés par les Bassins de la Garonne et de l'Adour) au $1 / 4$ de cette production (1), soit :

Totalité des Bassins de la Garonne et de l'Adour : 16.000 .000 de francs environ.

Voies navigables seules : 3.000 .000 de francs.

Ces chiffres paraissent bien modestes, quand nous venons de voir que le superdividende correspondant à la pèche des Migrateurs dans les mèmes cours d'eau navigables du Sud-Ouest s'élevait à 30.000 .000 francs avec plus de 650.000 kilogrammes de poissons de tout premier choix et plus de 2.000 kilogrammes de cáviar contrôlés.

L'importance des Migrateurs, qui ressort incontestable de cette comparaison, était encore bien plus considérable il y a seulement une trentaine d'années.

Pour le Saumon, il aurait fallu y ajouter la production des frayères aujourd'hui désertes du Gave-de-Pau, ainsi que celles de la Dordogne et de ses affluents (Vézère, Corrèze, Cère, Isle, Dronne).

Pour l'Alose, il faudrait envisager des prises dix fois supérieures à celles de ces dernières années. Un Département annonce à lui seul 500.000 kilogrammes. Des inscrits maritimes de l'Adour se souviennent avec regret de l'époque où ils prenaient, pendant deux mois de suite, 1 tonne d'alose par pêcherie et par jour, soit 60 tonnes dans la saison par emplacement autorisé de pêche à la senne.

L'Esturgeon, de son côté, a regressé dans des proportions inquiétantes à la suife des destructions importantes de jeunes dans les estuaires.

Nous sommes ainsi contraints de reconnaitre que, pour la seule région du Sud-Ouest, il nous a fallu payer les bienfaits de la civilisation (usines hydroélectriques, industries chimiques, egouts urbains...) d'une moinsvalue dans les pêches de Migrateurs atteignant, pour les trente dernières années, quelques millions de kilogrammes de poisson de premier choix, valant, au cours officiel actuel, une bonne centaine de millions de francs. En compensation, il est vrai, les industries installées sur les bords des cours d'eau du Sud-Ouest versent chaque année pour l'alevinage la somme fabuleuse de 150.000 francs en chiffres ronds...

Si nous nous reportions à un siècle en arrière, cette déchéance serait autrement importante, car ke Saumon fréquentait alors I'Adour, la Cha-

(1) Le nombre de Départements ne peut être retenu comme critérium acceptable de la répartition de la production. On peut, par contre, adopter grosso modo comme critérium facile la longueur navigable de l'axe fluvial principal de nos grands bassins :

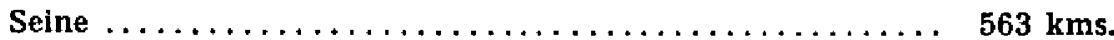

Loire ................................ 825 -

Rhône [de Lyon à la mer (335)], Saône (374) . . . . . . . . . 709 -

Garonne (462), Adour (134) . . . . . . . . . . . . . . . . . 596. 
rente, la Sèvre Niortaise et la Garonne, avec ses principaux aflluents : Ariège, Salat, Gers, Baïse, Lot, Truyère, Aveyron, Tarn, Agout...

Cette importance des Migrateurs désorientera vraisemblablement les pêcheurs et riverains actuels de nos rivières, elle n'étonnera pas outre mesure les habilués de la pêche maritime, qui savent bien que plus de la moitié du rendement de celle-ci est basé sur la seule capture des Morues, Harengs, Sardines, Thons et Maquereaux... migrateurs eux aussi.

Aux sceptiques qui ne voudraient pas admettre une déchéance de cette importance, nous demandons de bien vouloir réfléchir sur les quelques chiffres suivants :

En Alaska, les pêcheurs prirent, en 1910-1911, seules années dont nous ayons les chiffres de production, 35 millions et demi et 44 millions de Saumons (43.965.873 individus en registrés) (1).

Au Canada, en 1934, 169.700.000 livres de Saumong furent enregistrées par le Service des Pêcheries.

En Ecosse, il n'y a pas si longtemps, la Tweed, à elle seule, rapportait 170.000 Saumons.

En France, avant la révolution de 1789 , la pêche du Saumon pour les seuls fleuves côtiers bretons était affermée 200.000 livres or. Roule précise, par ailleurs, dans son "Traité raisonné de la Pisciculture et des Pêches ", que ces mémes cours d'eau bretons produisaient au xvil" siècle près de 4.000.000 de kilos de Saumon. - Le Comte de Pontcibaud, dont la Sioule, qui n'a plus aucun Saumon aujourd'hui, bordait le chateau, recevait une redevance annuelle de 150 Saumons. - En 1841, il était encore vendu à Quimperlé pour 40.000 francs de Saumon, soit, au cours de l'époque, 10.000 Saumons. Dans notre $\mathrm{xx}^{*}$ siècle de lumière, la production de Saumons doit être estimée d'après Kreitmann à :

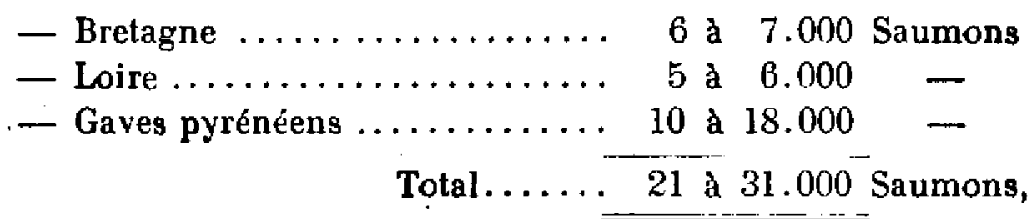

dont, il n'y a que cent ans, la seule Laîta Bretonne arrivait à produire le tiers ou la moitié.

De 1923 à 1934, les prises déclarées par les adjudicataires des dix filets barrages situés sur la Loire, dans le seul département de Maine-et-Loire, ont donné une moyenne annuelle de :

-60.300 kilos d Alose.

- 4.500 kilos d'Alose finte.

- 6.980 kilos de Saumon.

(1) Un ouvrage américain nous apprend que l'année 1917 a vu la prise de 70 millions de Saumons. 
Il serait souhaitable que les Services intéressés ne perdissent pas de vue ces considérations, et tinssent compte à l'avenir que, sur nos cours d'cau fréquentés par les grands migrateurs, l'évaluation des dommages piscicoles à l aide des formules du Prolesseur Lécen ne fait entrer en jeu que l'un des facteurs du problème, et de loin le moins important.

\section{III. - Raréfaction des mighateuns. - Ciauses et hemèdes \\ 1. - Causes passées et actuelles.}

Parmi ces causes, qui ont déjà fait l'objet de nombretses études, nous n examinerons rapidement que les trois principales.

a) Destruction par braconnage ou captures abusives. - Dans les estuaires, les captures par les Inscrits Maritimes qui n observent mème pas une réglementation déjà trop clémente à leur égard sont nettement abusives, particulièrement pour les Saumons adultes et les jeunes Esturgeons. Sur le cours moyen de nos cours d'eau, il en est de mème des prises faites aux barrages fixes ou aux filets trainants. Dans les hautes vallées, le braconnage du Saumon sur ses frayères est porté à la hauteur d'une institution.

Le remède est extrêmement simple en théorie, il suffit d'avoir des gardespêche nombreux et actifs. Dans la pratique, il nous faudra attendre encore quelques années pour que le nouveau corps des gárdes-pèche départementaux parvienne à un eflectif lui permettant de neutraliser suffisamment l'action des braconniers. Pour la première fois, en Décembre 1943, une vingtaine de gardes ont pu ètre rassemblés sur les frayères des gaves pyrénéens, le résultat a été des plus encourageants, le Saumon ayant pu, pour une fois, se reproduire sans payer un trop lourd tribut aux braconniers.

b) Edification de barrages ne permetlant pas aux mignateurs de parvenir sur leurs frayères. - Ces obstacles sont particulièrement à craindre pour le Saumon qui doit frayer dans les hautes vallées. La Commission Interministérielle des Barrages a heureusement décidé, dans ses séances des 2 Juillet 1927 et 26 Mars 1929, qu'aucune concession ne serait plus accordće sur nos plus riches cours d eau à Saumon (Aulne, - Ellé, - Allier, Adour en partie, - Gave d'Oloron, - Gave de Mauléon en partie - et Nive) et qu'une entente préalable entre le Service hydraulique et celui de la Pêche serait nécessaire pour les concessions visant : - la Canche, la Sienne, - la Sée, - La Sélune, - l'Elorn, - le Scorf et - le Gave de Pau.

Quant aux barrages construits sur ces rivières et ayant existence légale, on ne peut évidemment les supprimer. Reste le problème très délicat de les aménager par la construction d'échelles à Saumons quand cela est possible.

c) Pollution des eaux. - Tous les pècheurs connaissent, ou, tout au moins, ont la prétention de connaitre le problème posé par les déversements d'eaux résiduaires des industries et des grands égouts urbains. Le 
remède est ici particulièrement délicat et nulle part ailleurs le dicton " mieux vaut prévenir que guérir " n est mieux à sa place.

L'Administration est pratiquement désarmée devant les pollutions " chroniques ", les plus fréquentes, des installations qui ont une existence légale. Le problème réside donc dans l'imposition préalable aux inclustriels ou aux municipalités de conditions minima d'épuration; ces conditions pouvant, d'ailleurs, varier avec les caractéristiques des cours d'eau.

\section{II. - Cause nouvelle à prévoir.}

Fonctionnement par éclusées des usines hydroélectriques. - Les usines hydroélectriques peuvent connaître deux modes de fónctionnement. Elles peuvent, et c est un cas fréquent, adopter la marche dite "au fil de l'eau", c'est-à-dire où les turbines n'absorbent à aucun moment un débit supé. rieur à celui de la rivière. Le plan deau de la retenue amont ne descend donc jamais en dessous de la crête du barrage. Mais il y a de nombreux cas où la puissance nécessaire aux réseaux alimentés par l'usine ne reste pas constante au cours d'une mème journée. Il y aura des heures creuses et des heures de pointe. On y parvient en adoptant la " marche par éclusées ". Pepdant les heures creuses, les vannes sont réglées de façon à ne laisser passer que le débit minimum imposé. On constitue de la sorte dans Ia rełenue du barrage une réserve d'eau, qui permettra aux heures de pointe de faire passer par les turbines un débit massif très supérieur au débit moyen du cours d'eau.

L'usine fonctionnant "par éclusées " provoque à l'amont, dans son lac de retenue, des variations périodiques de niveau qui diminuent ou annulent, suivant leur amplitude, la production piscicole. Lors d'une étude sur I’Ain (1) nous avons montré qu'à l'aval, les à-coups provoqués par les éclusées transformaient le lit des rivières, le nivelaient, diminuaient considérablement la capacité biogénique, comblaient les gites des poissons et, par les hausses el baisses continuelles qu'elles provoquaient, créaient des conditions de vie presque impossibles pour le frai et l'alevin.

Nous demandions, en conséquence, qu'un bassin de compensation fût rendu oblirraloire. Nous sutrgérions de ne pas construire en ordre dispersé une dizaine d'usines sur des rivières différentes qui seraient toutes sacrifiées pour la pèche, mais de les grouper autant que possible sur une même rivière et de les soumettre à une réglementation judicieuse "d'exploitation concertée " de manière à obtenir un débit régularisé à l'aval du dernier barrage.

Bien que provenant d'un profane en matière hydroélectrique, ces vues n'étaient pas irréalisables, puisque nous voyons aujourd'hui une entreprise importante proposer un aménagement par "cycle journalier " de la

(1) R. Vibert. - Répercussions piscicoles du fonctionnement par éclusée des usines hydro-électriques. - Voir Bulletin - Janvier Février 1939, p. 109, - no 117 , Mars-Avril 1931, p. 137. 
Garonne en amont de Toulouse, entre Palaminy et Saubens et même jusqu'au Portet (1).

La chute de 90 mètres serait fractionnée en six à sept usines fonctionnant en principe simultanément avec le même débit. Le dernier barrage, compensateur en principe, procéderait à la redistribution avec des débits variables entre 240 mètres cubes seconde (débit pour lequel sont équipées les usines) et 90 mètres cubes seconde (débit exigé plus à l'aval par la Régie de Toulouse et les moulins du Bazacle), sauf le cas évidemment de crues dépassant 240 mètres cubes seconde. Le cube d'eau évacué à l'aval en 24 heures par ces débits variables serait égal au cube d'eau reçu à l'amont, d'où le nom d'aménagement par " cycle journalier ".

Un tel aménagement serait susceptible de produire par mètre de chute (2).

- Energie à puissance garantie distribuée pendant les heures appréciées (énergie de pointe)................... 4.200.000 Kwh.

- Energie à puissance variable distribuée pendant les autres heures $\ldots \ldots \ldots \ldots \ldots \ldots \ldots \ldots \ldots \ldots \ldots \ldots .4 .000 .000$ -

$$
\text { Soit un total de..... } 8.200 .000 \mathrm{Kwh} \text {. }
$$

alors qu'un aménagement au fil de l'eau ne produirait que $6.200 .000 \mathrm{Kwh}$.

Ce $n$ 'est qu'avec satisfaction que nous pouvons voir envisager la réalisation de telles expluitations groupées, que nous avions prôné nous-mème en 1939 ; les dégâts piscicoles qu'elles occasionnent étant très inlérieurs à ceux que produiraient un même nombre d'usines indépendantes, fonctionnant par éclusées et donnant la mème puissance totale. Dans le cas particulier de l'aménagement du secteur de Palaminy-Saubens, sur la Garonne, fleuve à grands Migrateurs type, quelques observations capitales s'imposent néanmoins :

$1^{\circ}$ Cette installation va transformer 60 kilomètres de Garonne, situés à proximité immédiate de Toulouse, en six à sept bassins successifs à niveau variable, dont la valeur piscicole va être en grande partie anéantie. Le cơté social de la question, s'il ne peut être considéré comme primordial, ne peut cependant ètre tenu pour négligeable alors que la seule ville de Toulouse comprend plus de 20.000 pêcheurs acquittant leur " taxe piscicole ";

$2^{\circ}$ Ce secteur de la Garonne compris entre Toulouse et Saint-Martory, avec ses affluents : l'A riège, - le Salat et l'Arize, était autrefois très riche en Saumons. Depuis la mise au point d'échelles à Saumons efficaces qui ont permis la réacclimatation de ce poisson dans les Gaves d'Aspe et d'Ossau, le Service de la Pèche escomptait pouvoir, après les hostilités, faire construire de telles échelles sur les barrages de Toulouse (Bazacle, Ramier-du-Château, - Cavaletade) et ramener aingi le Saumon dans cette

(1) G. Haelling. - L'énergie électrique contraint-elle l'homme au travail de nuit ? Electricilé - no4 107 et 108 ; Mars et Avril 1944.

(2) Calculs faits sur les débits de l'année 1939. 
région. Si l'équipement hydroélectrique de ce secteur de Garonne devait voir le jour, tous ces beaux projets seraient réduits à néant ;

$3^{\circ}$ Enfin, le projet ne prévoit qu une redistribution partielle des débits avec minimum de 90 mètres cubes seconde. Le bassin de redistribution du Portet évacuera donc à volonté tous les débits compris entre 90 et 240 mètres cubes seconde, et aura la faculté, tant que le débit de la Garonne n atteindra par 240 mètres cubes seconde, de faire varier en gros du simple au triple le débit du heuve à l'aval. Si l'on autorise de telles conditions de marche, il n'y aura pas compensation et nous aurons à l'aval du Portet des "éclusées " dont nous avons déjả dénoncé les ravages. Beaucoup plus a l'aval nous aurons évidemment les apports de débit du Tarn et du Lot qui peuvent ètre fort importants, mais pour peu que les installations en projet sur ces rivières revendiquent elles aussi la possibilité de marche par éclusées, c'est le lleuve entier qui risque d'ètre transformé en canal de chasse d'usine, jusqu'au point où se font sentir les marées de I'Océan (environ de Langon).

Il faudra alors tenir comple, ce qui n'a pas encore été fait, qu'un tel équipement hydroélectrique sera susceptible de produire des dégáts piscicoles non seulement dans ses eñvirons plus ou moins immédiats, mais que son fonctionnement par éclusées, s'il est autorisé, risque de porter un coup très sérieux aux Migrateurs qừ, de Langon à Toulouse, pourraient fort bien ne plus trouver les conditions nécessaires à leur reproduction. Il est, à l'heure actuelle, impossible de faire à ce sujet une estimation des dommages à envisager. Nous avons vu que les dégâts déjả imputables à l'industrialisation de nos rivières du Sud-Ouest, pendant ces trente dernières années, peuvent être évalués en chiffre rond à quelques cent millions de francs pour les seuls poissons migrateurs. Cette simple considération devrait inciter les Pouvoirs Publics à se montrer prudents, le jour où ils seront officiellement saisis de demandes de concessions semblables sur nos cours d'eau fréquentés par nos grands Migrateurs.

\section{III. - Le repeuplement artificiel : remedde polyvalent.}

Le repeuplement artificiel, qui passa pendant longtemps pour la clef de voute de la pisciculture moderne, peut-il être considéré comme le grand et principal remède permeltant de lutter elficacement contre ces diverses causes de raréfaction des Migrateurs 3 Il faut ici se garder de tout optimisme inconsidéré, pour chaque espèce intervient en effet une question de tech. nique opératoire et une question de rendement.

Pour le Saumon, la technique opératoire de la fécondation artificielle est bien au point, comme pour la Truite. Mais, si l'on en croit certains pisciculteurs américains et néozélandais, le rendement de la fécondation naturelle chez le Saumon égalerait la meilleure fécondation artificielle et supprime. rait tout l'intérêt de cette dernière, en dehors des problèmes d'acclimatation ou de réacclimatation de l'espèce dans les rivières qui en sont dépour. 
vues. La question est d'importance, espérons qu'elle pourra ètre un jour mise au point pour nos Saumons français.

Pour l'Esturgeon, quelques réussites isolées de fécondation artificielle sont à noter en U. R. S. S., en Allemagne et en Amérique. Les circonstances ne nous ont pas encore permis de nous attaquer à ce problème en mettant à contribution les frayères d'Esturgeons de la région de Varmande, sur la Garonne. I notre connaissance, aucune étude n'a été faite sur le rendement comparé des fécondations naturelles et artificielles de ce poisson. Nous ignorons s'il y a avantage à pratiquer cette dernière là où elle est possible.

Pour $]^{\cdot}$ llose, la fécondation artificielle a été praticquée sur une très vaste échelle aux Etats-Lnis et avec un magnifique succès, puisque la richesse en Nluse de bon nombre de rivières de la côte allantique a été augmentéc dians de très fortes proportions, ef que les Aloses pullulent dans les fleuves de la côte pacifique, de l'Alaska à la Californie, où elles étaient totalement iuconnues avant 1880. La fécondation artificielle paraît donc être pour ce poisson d'un rendement très supéricur à la fécondation naturelle ; malheureusement, toutes les tentatives faites en France jusqu'ici ont abouti à des échecs pratiques (1). La fécondation elle-même élait bien obtenue, mais les Aloses capturées ne donnaient jamais qu'une quantité infime d'œufs * maturitri. Etant donné l'intérèt tout particulier du repeuplement artificiel en Alose, il serait utile de rechercher les causes de ce comportement différent entre les Aloses américaines et françaises, de mème espèce cependant, les unes donnant facilement leurs ceufs en nombre considérable, les autres ne laissant entre les mains de l'opérateur qu'un très petit nombre d'œufs susceptible de fécondation.

Du repeuplement artificiel, il faut retenir que, pour certaines espèces, il peut être un appoint très appréciable a la reproduction naturelle, que dans le cas de rivières polluées ou rendues impropres à la vie du poisson par les éclusées qui y règnent, ce serait folie d'y déverser des alevins voués à la mort, que ce serait folie également de vouloir, par gout du moindre effort, axer une politique de rénovation de la pêche uniquement sur la piscirulture artificielle.

Il est essentiel de ne pas perdre de vue qu'il faut d'abord maintenir les rivières en conditions telles que les Poissons puissent s'y reproduire naturellement, ce qui malheureusement conduit à imposer une discipline désagréable aux industriels... et aux pêcheurs.

(1) Leclerc. - Note sur des essaís de multiplication artificielle de l'Alose dans le bassin de la Loire. - Voir Bulletin, no 123, Juin-Décembre 1941. 
CONCLUSIONS

Les textes législatifs que nous possédons pour imposer cette discipline ne sont évidemment pas parfaits, encore faudrait-il vouloir s'en servir.

Au fond, toute la sauvegarde de nos migrateurs se résume en un problème d'autorité :

Ou bien nous continuerons à fermer les yeux ou à faire appliquer pour la forme des sanctions anodines aux indisciplinés, qu'ils soient industriels ou braconniers, il y a alors toutes les chances pour que le rendement de la pêche de nos Poissons migrateurs continue à baisser d'année en année.

Ou bien, devant l'importance qu'avait et que peut reprendre en partie cette " industrie ", par une vigoureuse réaction, nous enjoịndrons aux divers Services intéressés d'exiger le respect des textes réglementaires pris dans le but de sauvegarder une richesse nationale. De grands espoirs peuvent alors être permis. 